\title{
Author Correction: Inhibiting PD-L1 palmitoylation enhances T-cell immune responses against tumours
}

Han Yao iD, Jiang Lan, Chushu Li, Hubing Shi, Jean-Philippe Brosseau, Huanbin Wang, Haojie Lu, Caiyun Fang, Yao Zhang, Lunxi Liang, Xiaolin Zhou, Chaojun Wang, Yu Xue, Yun Cui and Jie Xu (D)

Correction to: Nature Biomedical Engineering https://doi.org/10.1038/s41551-019-0375-6, published online 25 March 2019.

In the version of this Article originally published, 'palmitoyltransferase ZDHHC3 (DHHC3)' was incorrectly referred to as an 'acetyltransferase' rather than an as an 'acyltransferase'; this has now been corrected in five instances. In Fig. 3a, the label for the bottom row of the blots was mistakenly written as 'GAPHD'; it should have read 'GAPDH'. In the two right-most panels of Fig. $4 \mathrm{j}$, the antibody labels ' $\alpha$-PD-L1' for the reciprocal co-immunoprecipitation of DHHC3 were incorrect; they should have been ' $\alpha$-DHHC3'. These errors have been corrected in all versions of the Article. 